\title{
Die Semantik des ,guten Sterbens“ aus ethischer Perspektive
}

\section{Christof Breitsameter}

Eingegangen: 25. September 2019 / Angenommen: 27. April 2020 / Online publiziert: 4. Juni 2020 (C) Der/die Autor(en) 2020

Zusammenfassung Der Beitrag will der Frage nachgehen, ob innerhalb von Hospizen und Palliativstationen in Bezug auf typische Merkmale des „,guten Sterbens“ jenseits von in der Literatur erhobenen Konsensen auch Dissense beobachtet werden können, wie Sterbeverläufe, die als nicht gut betrachtet werden, zu Wort kommen, und schließlich: welche ethisch beachtlichen normativen Konsequenzen sich daraus ergeben.

Die Studie wird auf der Grundlage einer qualitativen Interviewstudie in der Befragung unterschiedlicher Akteursgruppen sowie im Vergleich dieser Gruppen durchgeführt.

Artikuliert werden - auch dissentierende - Vorstellungen von einem guten und einem schlechten Sterben, und zwar kaum von den Betroffenen und ihren Angehörigen selbst als vielmehr von den professionellen Akteuren. Die Ausprägung einer solchen Semantik hängt mit den Erfordernissen einer Praxis zusammen, die offenbar ein Rechenschaftsbedürfnis in Form von kommunikativer Vergewisserung evoziert.

Eine abstrakte Bestimmung des ,guten Sterbens“ (typischerweise: ruhig, selbstbestimmt, begleitet und gelassen) steht in Gefahr, die kontextuellen Besonderheiten der damit verbundenen Praxis nicht mehr in den Blick zu bekommen, was zu in ethischer Hinsicht problematischen Situationen führen kann.

Schlüsselwörter Guter Tod · Gutes Sterben · Ruhig · Bewusst · Selbstbestimmt · Begleitet · Gelassen 


\title{
The semantics of "good dying" from an ethical perspective
}

\begin{abstract}
Purpose The paper aims to investigate whether dissent-within hospices and palliative care units - can be observed with regard to typical characteristics of "good dying" (beyond the consensus that can be found in the literature), how statements about dying processes that are not perceived as good are expressed, and finally which ethically significant normative consequences arise from this.

Method The study is conducted on the basis of a qualitative interview study in different groups of actors and the relationships of these groups are compared with each other.

Results Notions of good and bad dying are articulated - even dissented upon-not so much by the patients and their relatives themselves as by the professional actors. This is connected with the requirements of a practice, which evidently evokes a need for accountability, namely for communicative assurance.

Conclusion An abstract designation of "good dying" (typically: quiet, self-determined, accompanied and peaceful) is in danger of losing sight of the contextual characteristics of the practice associated with it, which can lead to situations that are problematic from an ethical viewpoint.
\end{abstract}

Keywords Good death · Good dying · Quiet · Conscious · Self-determined · Accompanied $\cdot$ Peaceful

\section{Einleitung und Problemstellung}

In den letzten Jahrzehnten wurde immer wieder über den Begriff des guten Todes (besser müsste es heißen: des guten Sterbens) geforscht, häufig gestützt auf qualitative und quantitative Methoden (Miyashita et al. 2007; Morell 2012; Walters 2004). Dabei wurde der Begriff ,good death“ auch durch Begriffe wie ,appropriate, healthy, happy, peaceful“ oder „smoothy death“ spezifiziert (Mak und Clinton 1999). Versucht wurde mit diesen Studien, Plausibilitäten für einen übergreifenden, auch normativ wirkungsvollen Konsens in der Bestimmung eines guten Todes herzustellen. Die Ergebnisse sind intuitiv nachvollziehbar. Zum Teil wird die bedürfnisund wunschorientierte Begleitung Sterbender in Hospizen und Palliativstationen von dem einsamen, ohne Zuspruch und Anteilnahme sich vollziehenden Sterben in Krankenhäusern abgehoben, deren medizinische und pflegerische Routinen den Bedürfnissen und Wünschen von Sterbenden wie Angehörigen gegenüber unempfindlich sind: Hospize und Palliativstationen fördern ein ruhiges, selbstbestimmtes, begleitetes und gelassenes Abschiednehmen vom Leben sowie der sozialen Umgebung, sie suchen ein Sterben in Einsamkeit zu verhindern und beziehen sich in intensiver Weise auf letzte Konsumbedürfnisse und Erfahrungswünsche (Göckenjan und Dreßke 2002; Göckenjan 2008; Seale et al. 1997). Zum Teil versuchen Studien, einen plausiblen Konsens in der Bestimmung eines guten Sterbens durch eine Erhebung unverzichtbarer Merkmale in der Sterbebegleitung herzustellen (Steinhauser et al. 2000; Granda-Cameron und Houldin 2012). Ist das Sterben in Hospizen und Pal- 
liativstationen jedoch durch die Herstellung der beschriebenen Merkmale als gut zu beschreiben? Gelingt tatsächlich, was in idealen Vorstellungen beschrieben wird? Drohen in dem Versuch, einen Konsens herzustellen, die Dissense übersehen zu werden, die ja in der Kommunikation über den guten Tod bzw. das gute Sterben kaum verwundern können? Und wie werden Sterbeverläufe charakterisiert, die nicht als gut wahrgenommen werden?

Innerhalb des Projekts „Was ist ,gutes Sterben“?“ gingen wir der Vermutung nach, dass es neben Vorstellungen von einem guten auch Vorstellungen von einem schlechten Sterben gibt. Wir gingen auch davon aus, dass diese Vorstellungen voneinander abweichen können, je nachdem, welchen sozialen oder zeitlichen Raum sie einnehmen: Der soziale Raum bezieht sich auf Positionen im näheren oder weiteren Umfeld, insbesondere auf Ärzte, Pflegekräfte, Hospizhelfer, Seelsorger oder Angehörige, der zeitliche Raum egal welcher Dauer darauf, ob und wie sich die gegenwärtige Vorstellung von einem guten oder schlechten Sterben auf das in der Vergangenheit gelebte oder das für die Zukunft erhoffte Leben bezieht. Dabei war es Ziel unserer Studie, Positionsdifferenzen in zweifacher Hinsicht zu untersuchen: Einmal in der Befragung unterschiedlicher Akteursgruppen und dann in der Beobachtung der Äußerungen dieser Gruppen im Vergleich zueinander. Bei der Auswertung der Interviews bezogen wir uns zunächst auf diejenigen Stellen, an denen explizit über ,gutes“ oder „schlechtes“ Sterben bzw. über gute oder schlechte Sterbeverläufe gesprochen wurde, sei es, dass eine entsprechende Frage eine Antwort evozierte, sei es, dass die Gesprächspartner, insbesondere die Akteure im palliativen oder hospizlichen Rahmen, von sich aus darauf zu sprechen kamen. Erst im nächsten Schritt wurde das Umfeld solcher Aussagen mit einbezogen, und zwar zum einen benachbarte und zum anderen verwandte Aussagen, solche also, die sich, wenn man so will, implizit auf die Thematik bezogen.

\section{Methoden}

Nachdem die zuständige Ethikkommission nach Vorlage des Studienprotokolls die Studie für ethisch unbedenklich erklärt hatte ${ }^{1}$, führten wir von 2018 bis 2019148 Interviews mit Patienten/Bewohnern (22) von Hospizen und Palliativstationen, ihren An- und Zugehörigen (14) sowie mit Ehrenamtlichen (16), Pflegekräften (42), Sozialarbeitern (7), Seelsorgern (8), Therapeuten (15), Hospizleitungen, Verwaltungskräften und Hauswirtschaftern (8) und Ärzten (16) durch, die in Hospizen und auf Palliativstationen tätig sind. Die Auswahl der Studienteilnehmer erfolgte durch Kontaktaufnahme zu Hospizen, Palliativnetzwerken und Palliativstationen von Krankenhäusern in Baden-Württemberg, Bayern, Hamburg und Sachsen-Anhalt. Insgesamt forschten wir in fünf Hospizen und auf zwei Palliativstationen.

Die Gespräche fanden in den Kliniken oder in den Hospizen statt. Sie orientierten sich an thematischen Leitfäden und erfolgten in halbstandardisierter Form. Die Leitfäden waren für die unterschiedlichen Personengruppen konzipiert: Es gab jeweils einen Leitfaden für die Bewohner und Patienten, für die An- und Zugehörigen,

\footnotetext{
1 Ethikkommission bei der Medizinischen Fakultät der LMU München, 30.11.2017, Projekt Nr.: 17-684.
} 
für das Personal und einen für die Ärzte. Patienten und Bewohner wurden danach gefragt, warum sie ins Hospiz bzw. auf die Palliativstation gekommen sind, wie sie die Institution wahrnehmen, ob sie ihnen gefällt, wie sich ihr Kontakt zu Ehrenamtlichen, Pflegekräften, Therapeuten, Seelsorgern und Ärzten gestaltet und welche Wünsche sie haben. Die An- und Zugehörigen wurden danach befragt, warum ihr An- bzw. Zugehöriger nun im Hospiz oder auf der Palliativstation ist, ob es ihm dort gefällt, welche Wünsche er äußerst, wie sie die Institution wahrnehmen und ob und wie sie Kontakt zum Personal haben. Das Personal fragten wir nach der Gestaltung des Alltags, nach den Kontaktmöglichkeiten mit den Patienten/Bewohnern und Anund Zugehörigen, nach den Bedürfnissen und Bedarfen der Patienten/Bewohner sowie nach gelungenen und problematischen (Sterbe-)Begleitungen. Die Ärzte fragten wir nach Gestaltungsräumen der alltäglichen Praxis, den Beziehungen zu den Patienten/Bewohnern, ihren Wünschen und Ängsten, nach den Beziehungen zu den Pflegekräften und Angehörigen sowie nach gelungenen als auch problematischen (Sterbe-)Begleitungen.

Die Datenerhebung und die Auswertung orientierten sich im Wesentlichen an den Methoden der von Barney Glaser und Anselm Strauss (Glaser und Strauss 1967) begründeten und von Juliet Corbin und Anselm Strauss (Corbin und Strauss 2008) modifizierten Form der ,grounded theory“. Die im Forschungsprozess gewonnenen Kategorien wurden hinsichtlich ihrer theoretischen Eigenschaften ausgearbeitet und wiederum im Feld überprüft. Der Prozess des Kodierens wurde von den beteiligten Forschern, welche einen unterschiedlichen fachlichen Hintergrund haben, unabhängig voneinander durchlaufen. Die Ergebnisse wurden verglichen und in regelmäBigen Besprechungen diskutiert. Der Prozess der Datenerhebung wurde beendet, nachdem aus den Interviews keine zusätzlichen Kategorien mehr gewonnen werden konnten, sodass wir in Bezug auf die zugrunde liegenden Forschungsfragen von einer theoretischen Sättigung ausgehen. Die Interviews wurden nach Aufklärung und schriftlicher Einverständniserklärung der teilnehmenden Personen mit einem digitalen Aufnahmegerät aufgezeichnet und im Anschluss vollständig wörtlich transkribiert, wobei auch eine Anonymisierung der Daten erfolgte. Bei der Transkription bedienten wir uns des Computerprogramms f4transcript und zur Analyse nutzen wir das Programm MAXQDA.

\section{Empirischer Befund}

Im Folgenden wird die Zuschreibung eines guten oder schlechten Sterbens getrennt nach denjenigen Akteuren in einem Hospiz oder einer Palliativstation betrachtet, die sich zu diesem Thema explizit oder implizit äußern. Überschlagsmäßig lässt sich sagen, dass typische Merkmale des guten Sterbens in ähnlicher Form auch bei Interviewpartnern benannt werden, die wir nicht zitieren. Zitiert werden die prägnantesten Beschreibungen dieser Typisierungen. Merkmale von Verläufen, die als nicht gelungen (etwa als herausfordernd oder konflikthaft) dargestellt werden, stellen dagegen in der Regel Einzeläußerungen dar. 


\section{Ärzte}

Ein Arzt reflektiert die Standpunktabhängigkeit einer Antwort auf die Frage ,Was ist gutes Sterben? Was ist gut? Aber vielleicht ist es eine ganz individuelle Frage. Was stelle ich mir zum Beispiel darunter vor, unter gutem Sterben? Und vielleicht ist das auch die Vorstellung, mit der ich auch zu den Patienten gehe. Natürlich frage ich die Patienten, was sie sich selbst darunter vorstellen, und ja, was ich tun kann, dass es ihren Vorstellungen entspricht.“ (E-A-P-5, 419-423) Damit wird zumindest die Möglichkeit einer Spannung zwischen dem, was der Arzt, und dem, was der Patient für gut hält, thematisiert. In den allermeisten Fällen stellen die Symptom- bzw. Schmerzkontrolle sowie die Begleitung der Sterbenden, die sich nicht alleingelassen fühlen sollen, die wichtigsten Bedingungen für einen ,ruhigen“ (EA-P-5, 442) Sterbeverlauf dar - fast identisch äußert sich ein anderer Arzt (E-AH-2, 321). Allerdings gibt es nicht selten Patienten, die ,zum Ende ihres Lebens nicht diese Ruhe entwickeln können, die oft so gut in der Begleitung ist, ja? Die zum Schluss psychisch dekompensieren, die psychiatrische Symptome entwickeln. Die auch ablehnend, aggressiv sind, die einfach von dem, zu denen man keinen Zugang findet. (...) Ja, weil das oft so anstrengend ist, wenn man bereit ist zu tun und zu geben und wenn man auf starke Ablehnung oder depressive Verstimmung, einfach komplette Ablehnung stößt, dann ist es oft sehr, sehr schwierig, aber passiert oft.“ (E-A-P-5, 913-931) Eine Spannung zwischen der Vorstellung des Arztes und der Vorstellung der Patienten von einem guten Sterben sei auch beim Thema „Suizid“ zu beobachten, weil ,immer mehr Menschen als gutes Sterben inzwischen das selbstbestimmte oder vermeintlich selbstbestimmte Sterben favorisieren (...), dass ich sage (...), ich bin autonomer Mensch, ich habe Selbstbestimmungsrecht, und da möchte ich auch selbstbestimmt sterben." (E-A-P-5, 461-465) In Wirklichkeit stecke dahinter jedoch die Angst vor Schmerzen oder die Angst, alleingelassen zu sein. (EA-P-5, 490-495) Eine palliative Sedierung hat nach Aussage des Arztes allein der Symptom- bzw. Schmerzlinderung zu dienen, wird jedoch nicht eingesetzt, wenn es darum geht, „,nur“ das Bewusstsein zu reduzieren, weil ,man irgendwas nicht mitbekommen will“ (E-A-P-5, 463). Überhaupt bezeichnet dieser Arzt die Rede vom „guten Sterben“ als „Semantik“: „Also, ich behaupte: Nein, das gibt es nicht.“ (EA-P-5, 1139).

Ein ruhiger Sterbeverlauf sei, so ein anderer Arzt, allerdings auch für das Pflegepersonal sowie für die Angehörigen wichtig: Ein Patient ist in der ,ganz präfinalen Phase, eigentlich war er bettlägerig (...) aufgestanden, hingefallen, Kopfplatzwunde, viel Blut, Unruhe, das ist natürlich für alle Beteiligten ziemlich unschön. Und grad auch wenn dann beispielsweise Angehörige dabei sind. Weil man vorher versucht (...) $\mathrm{zu}$ vermitteln, dass das Sterben gut begleitet sein kann und ruhig vonstattengehen kann und dann so das am Ende nochmal passiert, das ist dann schlecht (...), es ist natürlich nicht so, wie man sich das wünscht, für den Patienten, für die Angehörigen in erster Linie. Aber natürlich belastet das auch das Pflegepersonal, die dann auch direkt vor Ort natürlich sind, ne. Aber gut ist eben, find ich, wenn's ruhig ist, gelassen ist und eigentlich nach den Wünschen des Patienten, ja, abläuft, weil derjenige der ist, der stirbt." (E-A-H-2, 329-339) Dieser Arzt äußert sich auch zur Schmerzmedikation: ,Ja, es gibt immer mal wieder Patienten, die große, ich würde nicht sagen 
Angst haben, aber Vorbehalte zumindest auch gerade gegen Schmerzmittel. Weil die eben dämpfend wirken können, wie auch immer. Da versuch ich das dann aber schon im Vorwege eigentlich schon eben auch durch Gespräche zu erklären oder dass man eben den Schmerz nicht in Kauf nehmen muss, wenn sozusagen noch die Möglichkeit besteht, ein Schmerzmittel zu geben oder höher zu dosieren, damit eben diese Unruhe am Ende nicht reinkommt. Wenn es jemand ablehnt, muss man das natürlich akzeptieren, man kann ja nicht Schmerzmittel verabreichen, wenn, wenn der Patient das nicht wünscht oder auch gar ablehnt.“(E-A-H-2, 347-355).

Eine Ärztin sieht Grenzen der Selbstbestimmung, etwa bei der palliativen Sedierung, die nur der Symptom- bzw. Schmerzlinderung dienen darf, keineswegs dem Zweck, das Bewusstsein des Patienten - selbst auf dessen ausdrücklichen Wunsch hin - zu vermindern oder gar auszulöschen. (E-A-P-6, 448-463) Allerdings bringt eine palliative Sedierung erwünschte Folgen für das professionelle Umfeld mit sich, wenn nämlich ,die Patienten dann einfach auch unruhig sind, wo man dann auch eben so diese Gratwanderung zwischen Sedieren, aber doch noch irgendwie diese Persönlichkeitsrechte behalten, also diese Würde. Keiner von uns würde einfach wegsediert werden sollen, nur weil man unruhig ist. Man muss ja auch die Würde der Pflege auch irgendwie bewahren." (E-A-P-6, 769-774) Ein Arzt versucht, bei einer Sedierung das Bewusstsein möglichst zu erhalten, um mit dem Patienten kommunizieren zu können, also ein selbstbestimmtes Sterben zu ermöglichen, selbst wenn der Betreffende nur noch in Ruhe sterben will. (E-A-H-1, 655-665) Dennoch ist für diesen Arzt „,ein gutes Sterben ein ruhiges Sterben“ (E-A-H-1, 698 und 706), was vor allem über die Symptom- und Schmerzkontrolle erreicht werden kann, wobei es schwierige Fälle gibt, die ,schmerztechnisch kaum in den Griff zu kriegen sind“. (E-A-H-1, 770) Eine Ärztin sieht einerseits das Erfordernis, bei der Medikamentengabe das Bewusstsein des Patienten möglichst zu erhalten, ist jedoch bereit, eine Eintrübung des Bewusstseins in Kauf zu nehmen, um Unruhe zu lindern. (E-AH-7, 139-142, ähnlich E-A-H-7, 299-301) Der Konflikt zwischen Bewusstheit und Selbstbestimmtheit einerseits und deren Beeinträchtigung durch eine Schmerzmedikation andererseits wird durch schrittweises Einstellen der entsprechenden Dosis „gelöst“. (E-A-H-7, 324-339) Für einen anderen Arzt ist ,gutes Sterben“ ein friedliches Sterben (E-A-H-6, 213), wobei für ihn Unruhe häufig schwierig in Griff zu bekommen ist. (E-A-H-6, 262-263) Die Berücksichtigung des professionellen Umfelds bei der Bestimmung eines guten Sterbens wird noch deutlicher in Extremfällen, wo die Pflegekräfte durch eine ruhigstellende Medikamentengabe geschützt werden sollen: „Wir hatten eine Patientin, die hat halt dann wirklich das Zimmer mit ihrem Kot beschmiert, täglich. Und da muss man schon auch auf die Pflege achten. Und da so diese Gratwanderung zu sehen, welche Unruhe lässt man zu, welche nicht?“ (E-A-P-6, 774-777) Dieselbe Ärztin beschreibt einen guten Sterbeverlauf folgendermaßen: „Natürlich beschwerdearm, beschwerdefrei, wenn wir es schaffen, dass wir den Patienten, jetzt so wirklich medizinisch gesehen, dass wir die Symptome nehmen, also dass er da gut damit zurechtkommt. Und dann glaube ich schon, dass man einfach diesen Bedürfnissen sehr nahekommen muss, und eben die Patienten auch mal in Ruhe lassen muss. (...) Die letzten Wochen, Tage, Stunden, das ist einfach nur noch Ruhe. Die sind in sich zurückgezogen, brauchen nicht mehr viel. Und 
ich glaube, wenn man ihnen dann die Ruhe gibt, ist das ein guter Verlauf." (E-AP-6, 530-541).

Für eine Ärztin ist das ,,ruhige, friedliche“ (E-A-P-4, 170) Sterben ebenfalls professionstypisch an die gelungene Symptom- bzw. Schmerzkontrolle gebunden, die nicht immer einfach ist, etwa wenn sich eine Patientin weigert, Schmerzmittel zu nehmen: „Wenn die ihre Schmerzmittel genommen hätte, wäre die schmerzfrei gewesen. Aber sie war aufgrund ihrer Psychostruktur so, dass man das jeden Tag mit Engelszungen mit ihr besprechen musste. Bis sie dann zum Schluss so somnolent war, dass man ihr einen Perfusor machen konnte, dann gab es eine gute Symptomkontrolle, dann ist sie auch friedlich gestorben, friedlich, also ruhig gestorben." (EA-P-4, 224-229) Hier wurde der Patientenwille lange Zeit berücksichtigt, am Ende freilich überstimmt. Die medizinische Maßnahme diente offenbar den Erwartungen des professionellen Umfelds an ein ruhiges, friedliches Sterben. Dass die Erwartungen an ein ruhiges Sterben auch vom Team her kommen, wird durchaus reflektiert: „Und was ich im Team beobachte, ist, dass das Bedürfnis, dass jemand ruhig stirbt, zunimmt. Also dieses Wissen, dass man, wenn man sein Leben lang gekämpft hat, auch einmal im Kampf stirbt, das ist zwar da, aber es wird immer schwerer auszuhalten.“ (E-A-P-4, 355-358) Diese Ärztin ist der Meinung, dass ,die Pflege mehr das Bedürfnis hat, dass jemand ruhig, friedlich“ stirbt. (E-A-P-4, 423) Offenbar kann die Enttäuschung von Erwartungen an einen gelungenen Sterbeverlauf an den Eindruck persönlichen bzw. professionellen Versagens gekoppelt sein, wie die Ärztin bemerkt: „Ich glaube, es sind zwei Dinge wichtig, oder zwei Dinge, die schwer verarbeitet werden. Das eine ist, wenn wir jemanden tot auffinden. Das ist immer so ein stückweit, wir waren nicht da. (...) Und das andere ist eben, wenn jemand zum Schluss noch gekämpft hat, also unruhig war, gerufen hat, geschrien hat, solche Dinge. Zum Beispiel, es wird lange nicht so memoriert, wenn jemand mit Atemnot gestorben ist, weil da können wir etwas tun." (E-A-P-4, 422-446) Deutlich zeigt sich hier ein Rechtfertigungsbedarf, der professionelles Handeln evoziert und ein Nicht-Handeln eher unter Verdacht stellt.

\section{Pflegekräfte und Hospizhelfer}

Von einer Pflegekraft wird die Schmerz- und Symptomkontrolle als Voraussetzung für ein ruhiges und damit gutes Sterben benannt. (E-PF-H-7, 400-403, ebenso E-PFH-5, 131 oder E-PF-H-28, 574) Gleichzeitig ist ihr bewusst, dass es nicht einfach darum geht, Unruhe zu ,dämpfen“ (E-PF-H-7, 499), sondern genauer hinzuschauen, woher die Unruhe eines Patienten konkret kommt und wie sie behandelt werden sollte. (E-PF-H-7, 500-503) Einer Pflegekraft ist es wichtig zu wissen, ob das Sterben ruhig oder unruhig vonstattenging: Schwierig ist es, wenn jemand ,einsam gestorben ist und keiner hat gesehen, war es ein ruhiges Sterben, hat sie vielleicht vorher eine Unruhe gehabt““. (E-PF-P-6, 47-48) Offenbar widerspricht Unruhe der Vorstellung von einem guten Sterbeverlauf: „Das ist auch ein Symptom, diese Unruhe, und oft wissen wir bei dieser Unruhe, dass es sich um eine präfinale Unruhe handelt, und dann geben wir Bedarf, und dann kommen die auch ganz schnell in eine Ruhe. Und da sind wir auch sehr großzügig. Also wenn wir jetzt einen Bedarf geben und der ist nicht nach zehn Minuten ruhig, dann geben wir direkt wieder etwas. Also wir 
dürfen Bedarf direkt schnell wieder noch einmal geben." (E-PF-P-6, 150-155) In dieser Aussage bleibt unklar, ob der Wille der Patienten Berücksichtigung findet, wenn Beruhigungsmittel verabreicht werden, bzw. ob der Patientenwille überhaupt noch berücksichtigt werden kann, weil ja kaum noch Kommunikation stattfindet. Beruhigungsmittel werden gerade mit dem Ziel verabreicht, dass der Verlauf ruhig und friedlich ist: „Oder jetzt kriegt er, weil er so schlecht ist, ein Beruhigungsmittel, aber wir wissen alle, dass er jetzt vielleicht nicht mehr in die Kommunikation geht. Dass er mehr schläft, aber uns ist wichtiger, er schläft und ist entspannt. Alle sind an einem milden Verlauf interessiert. Und dass ein Patient in Ruhe, ohne Schmerzen, ohne Unruhe sterben kann. Und möglichst noch mit Familie.“ (E-PF-P-6, 571-576) Hier werden offenbar Bedürfnisse im Umfeld des Patienten, also bei Pflegekräften und Angehörigen, handlungsleitend, die Bedürfnisse des Patienten treten hingegen zurück. In einem anderen Fall schildert die gleiche Pflegekraft jedoch auch, dass sie einen Patienten übernimmt, um seinen Willen, keine Schmerzmittel zu nehmen, erfüllen zu können, eine Entscheidung, die anderen Pflegekräften schwer fällt, weil sie ihre Auswirkungen nicht oder nur schwer ertragen können bzw. wollen: „Und da haben manchmal die Schwestern Probleme, weil wir haben ja die Anordnung, wir könnten alles Mögliche, wir dürften geben. Wir würden geben. Aber er hat seinen freien Willen, und ich kann dann eher einmal mit den Patienten und sage: ,Mensch, Leute, wenn du den nicht ertragen kannst, gib ihn mir. Weil ich biete ihm das an, er versteht mich, und wenn er sagt: nein, dann sagt er nein, aber wie gesagt, das muss man aushalten, dass er das nicht will.“ (E-PF-P-6, 580-594) Nicht alles ist eben „so nett zum Anschauen“ (E-PF-P-6, 602), manches muss man ,aushalten können“. (E-PF-P-6, 608) Ähnlich äußert sich eine andere Pflegekraft. Lehnen Patienten eine Symptombehandlung ab, weil sie ihre Schmerzen spüren wollen, fühlt sie sich „hilflos“ (E-PF-H-25, 305), doch respektiert sie die Autonomie der Betreffenden. (E-PF-H-25, 306-319) Gutes Sterben vollzieht sich für sie symptom- und schmerzfrei, die Patienten sollen mit dem Verlauf ,zufrieden“ sein können. (E-PF-H-25, 563) Allerdings geht das Sterben für sie manchmal zu langsam vor sich. (E-PFH-25, 572-579) Eine Pflegekraft äußert allerdings auch ihre Skepsis, die gelungene Schmerz- und Symptomkontrolle, die einen harmonischen Sterbeverlauf ermögliche, sei einfachhin Anzeichen für ein gutes Sterben: In bestimmten Situationen zeigen sich oft ganz andere, vor allem soziale Erfordernisse bzw. Bedürfnisse. (E-PF-H-4, 607-613) Teilweise wird ein ruhiger Sterbeverlauf stark idealisiert: „Und dann ist das so ein friedliches Sterben, wo ja so sehr irgendwie immer wieder dieser, die Magie in diesem Moment durchkommt, dieses, das Magische, Unfassbare, Unbeschreibliche und das kommt natürlich in einer friedlichen, ruhigen Atmosphäre mehr zur Geltung, wie wenn ich damit beschäftigt bin, jetzt gleichzeitig Blut abzusaugen und Spritzen zu geben und was weiß ich was. Wenn das in einer Ruhe verläuft, wo ich eigentlich nichts weiter tun muss, aber dabei sein darf." (E-PF-H-14, 52) Wo ein Sterben nicht friedlich verläuft, stößt die Pflegekraft nach eigener Auskunft ,,an Grenzen“ (E-PF-H-14, 18).

Teilweise besteht also die Tendenz, die Autonomie des Patienten zu überstimmen, teilweise halten es die Pflegekräfte auch aus, dass ein Patient keine Symptom- bzw. Schmerzbehandlung will. Eine Pflegekraft bemerkt, es sei schwierig, mit ,notorische(r) Unruhe“ (E-PF-H-22, 180) in der präfinalen Phase umzugehen, weil hier im 
Grunde eine „Eins-zu-Eins-Betreuung“ (E-PF-H-22, 186-187) erforderlich wäre, was kaum zu leisten ist. Obwohl die Schmerzsymptome für die Umgebung ,nicht mehr aushaltbar" (E-PF-H-22, 207-215) waren, wurde in einem Fall der Patientenwille möglichst lange respektiert, zumindest wurde erst ,ganz, ganz, ganz am Schluss Morphin angenommen“ (E-PF-H-22, 213). Auch in einem anderen Fall erfüllt sich der Wunsch der Pflegekraft, Patienten ,frei von physischen und psychischen Schmerzen“ (E-PF-H-11, 336-337) zu sehen, nicht, weshalb Schmerz- und Symptomfreiheit nicht Hand in Hand mit der Begleitung gehen, sondern ein Gegengewicht dazu bilden. Eine andere Pflegekraft bemerkt: „Oder wenn jemand, ja, seine Situation so überhaupt nicht mehr einschätzen kann, dann spritz ich ihm auch was, wenn er also, wenn ich das Gefühl hab, es ist vonnöten. Ich mein, klar, ich hab hier auch ne Bedarfsmedikation. Ich mach da jetzt nichts aus dem Leeren oder so, ja? Aber die Situation gibt's, ja, wo du dann, du kannst jemanden, der Luftnot hat und im Verlauf schon unterwegs ist, den kannst du nicht vorher immer fragen: Darf ich Ihnen jetzt Morphin spritzen? Ich sage zwar, was ich mache. Ne? Ich geh jetzt und hol Morphin, oder: Ich bin gleich wieder bei Ihnen. Ich seh, Sie haben Luftnot, ich spritz Ihnen jetzt was, ja? So würde ich das machen." (E-PF-H-23, 297-305) Hier ist das Gefühl als Gespür interpretiert, in einer Situation das Richtige zu tun und so den Patientenwillen zu erfüllen.

Hinter dem Wunsch mancher Patienten, Schmerzen auszuhalten, steckt offenbar eine bestimmte Vorstellung von Autonomie und Bewusstheit. Die Herausforderung für die Pflegekräfte besteht darin, Symptom- und Schmerzfreiheit im Gleichgewicht mit der Bewusstheit des Patienten zu halten. (E-PF-H-11, 436-438) Als schwierig wird empfunden, wenn man an den Willen des Patienten nicht mehr herankommt. (EPF-P-9, 350-353) Eine Pflegekraft versucht, Patienten, die ihre Schmerzen aushalten wollen, über Möglichkeiten der Schmerz- und Symptomkontrolle aufzuklären, akzeptiert jedoch letztlich den Willen der Betreffenden. (E-PF-H-30, 234-241) Das Ringen mit dem Patientenwillen klingt auch in der Bemerkung einer Pflegekraft an, die sich generell auf Patienten bezieht, die zwar starke Schmerzen haben, aber eine Schmerzmedikation ablehnen: „Das ist eine große Herausforderung. Aber ich hab es auch einmal schon erlebt, dass wir das respektieren mussten, weil der das ganz klar schon vorher gesagt hat. Und wirklich nur ganz, ganz, ganz am Schluss Morphin angenommen hat, und das war auch schwer auszuhalten für uns. Das haben wir mit dem Gast so besprochen, dass es für uns eigentlich nicht mehr aushaltbar ist. Dass wir uns das nicht mehr mit angucken können. Und trotzdem mussten wir es eine Zeitlang noch mitmachen." (E-PF-H-22, 210-216) Ein Hospizhelfer versucht, Autonomie und Fürsorge schrittweise dadurch miteinander zu verbinden, dass Patienten die Erfahrung vermittelt wird, durch eine Schmerzmedikation ein höheres Maß an Lebensqualität zu erlangen: „Und dann probieren sie es aus und merken, sie fühlen sich dann viel wohler, weil die Lebensqualität nimmt ja dann zu und man ist trotzdem präsent usw.“ (F-H-7, 157-163) Der Respekt vor der Autonomie der Patienten muss sich auf ganz unterschiedliche Verläufe einlassen, was die Pflegekräfte vor besondere Herausforderungen stellt: Patienten haben unterschiedliche Auffassungen vom guten Sterben. Manche wünschen einfach, schlafen und hinüberschlafen zu können, manche möchten ,,präsent“ bleiben und ihr Sterben „,mitverfolgen“ (EPF-H-19, 155) und „,reflektieren“ können. (E-PF-H-19, 157) Die Pflegekraft, die 
dies äußert, würde eher für Präsenz plädieren, freilich nicht so, dass jemand „dann Schmerzen aushalten muss“ (E-PF-H-19, 167). Doch wird ihrer Meinung nach die gängige Praxis zu wenig hinterfragt, ,es gibt immer die gleiche Bedarfsmedikation, die angewendet ist, das unterscheidet sich kaum. Und wir geben das dann. Weil das ist angeordnet. Bei Übelkeit geben wir das, und ob jemand dann darauf so reagiert, dass er zwölf Stunden schläft, und der andere schläft aber nur zwei Stunden, das, Hauptsache, die Übelkeit ist beseitigt.“ (E-PF-H-19, 169-172) Man müsste deshalb genauer hinschauen, was für jeden einzelnen Menschen in dem Moment wichtig ist. (E-PF-H-19, 174) Es besteht die Gefahr, dass sich „Routinen“ (E-PF-H-19, 174) einschleichen, die dem Patienten bzw. seinem individuellen Wohl nicht gerecht werden.

$\mathrm{Ob}$ der Verlauf gut oder schlecht war, ist standpunktabhängig und wird vom Gefühl, ob es gestimmt oder gepasst hat, beurteilt, wie eine Pflegekraft ausführt: „Also für mich ist das ganz häufig so, dass ich das mit meinem Gefühl irgendwie entscheide. Dass ich jetzt gar nicht sagen kann, zum Beispiel hatten wir jetzt eine Frau, die ist ganz plötzlich, also für uns alle ganz unerwartet, war sie einfach tot. Und da kann man jetzt sagen, ach, das war ja jetzt gut für sie, weil sie hatte so schreckliche Wunden, und sie musste gar nicht, also, das hat, war jetzt irgendwie gut. Aber für mich fühlt sich's gar nicht so gut an. Also, weiß ich nicht. Und dann gibt es andere Gäste, die sind wirklich präfinal und man hat so das Gefühl, es zieht sich alles wahnsinnig hin und trotzdem hab ich das Gefühl, am Ende hat es irgendwie dann doch gepasst, ja. (...) Es muss auch vom Gefühl stimmen, und es muss auch, man muss auch spüren, dass es für den Gast irgendwie gestimmt hat." (E-PF-H-19, 371-384) Dabei ist die Beurteilung des Verlaufs an die Möglichkeit von Kommunikation gekoppelt, wie eine andere Pflegekraft formuliert: „Aber dann die letztendliche Sterbephase, so der letzte Tag, oder die letzten zwei Tage, da sind die Gäste eigentlich in der Regel doch immer mehr für sich, ja. Und immer abgeschotteter. Und das belastet mich dann schon, weil man so viel erahnen muss: Was ist jetzt in der ganz, in der allerletzten Phase, aber die sich jetzt auch in der letzten Zeit hier bei unseren Gästen oft recht lang gestaltet. Dass sie dann wirklich in komatösem Zustand über mehrere Tage sind, wo alle dann nur warten: Wann passiert es endlich? Und dann so gut wie kein Zugang mehr zum Gast möglich ist, ja. Und ansonsten, genau, bis auf die letzte Phase würde ich viele Begleitungen als gelungen benennen." (E-PF-H-21, 314-324) Das Thema Autonomie spielt hier eine Rolle, gerade weil sie nicht geübt werden kann, doch ermöglicht werden soll.

Auffällig häufig wurde das gute Sterben bei Pflegekräften und Hospizhelfern mit der Vorstellung assoziiert, dass ein Mensch mit sich selbst ,im Reinen“ ist bzw. sein soll. In diesem Sinn äußert sich eine Hospizhelferin (F-H-1, 23), die den Sinn dieser Beschreibung durch den Kontrast deutlich zu machen versucht: Nicht mit sich im Reinen ist für sie ein Patient, der sich seiner Umwelt gegenüber in einer schwierigen Weise verhält, so etwa ,ein Mann, der einfach nicht sterben konnte. Der war nie böse zu mir, aber er war, ich nenne es mal, einfach ein bisschen ruppig. Und hatte keinerlei soziale Kontakte. Das hat mir gezeigt, der ist mit sich nicht im Reinen, der hat Angst, wahnsinnig Angst vor dem, was kommt.“ (F-H-1, 29) Häufig wird das „Mit-sich-im-Reinen-Sein“ mit einer gelassenen Haltung assoziiert: „Ich glaube (...), dann kann man leichter loslassen. “ (E-PF-P-4, 51) Wo das nicht gelingt, 
wird die Umgebung in Mitleidenschaft gezogen: „Die war mit sich und mit allem im Unreinen. Und war wirklich sehr, ja, hässlich zu allen. Oder zu vielen, ja?“ (F-H-2, 31) Doch wendet sich alles zum Guten: „Aber auch diese Dame wurde ganz am Ende weich." (F-H-2, 33) Die Pflegekräfte empfinden ein gutes Sterben häufig als ein Sterben in Frieden (E-PF-H-13, 50), wo ein Mensch sich ,hingeben“ und ,loslassen“ kann. (E-PF-H-14, 38) Teilweise werden erkennbar persönliche Ideale vom guten Sterben als Deutungsschlüssel eingesetzt, ohne dass diese Erste-Person-Perspektive reflektiert, geschweige denn mit einer Dritte-Person-Perspektive, die natürlich nur vermutet werden kann, in Kontakt gebracht wird: „Ich spür, wenn es dem Patienten gut geht, also wenn er seinen Weg geht und, ähm, ähm, ja, ob er jetzt im Kampf ist oder in die Befreiung oder in den Frieden hinein oder in ein, in ein, ja für mich ist immer wichtig, dass ein Ziel da ist, da, das find, das find ich ganz wichtig." (E-PF-H-17, 405-408) Stark idealisierend wirkt auch die Charakterisierung einer Pflegeschülerin: Von einem guten Sterben könne dann gesprochen werden, ,wenn der Patient friedlich gehen kann. Ohne Schmerzen, ohne irgendetwas bereuen zu müssen." (E-PF-H-18, 254-255) Umgekehrt liege keine gelungene Sterbebegleitung vor, ,wenn der Patient sich schlecht fühlt. Und auch nicht seinen Frieden damit nicht finden kann, wenn er nicht gehen möchte." (E-PF-H-18, 260-261) Gelegentlich wird die Standpunktabhängigkeit des Urteils darüber, was denn ein gutes oder schlechtes Sterben ausmacht, reflektiert: „Ich meine, da muss man schauen, wie man gut für sich selber definiert, ob es ein gutes Sterben ist. (...) Das gute Sterben für uns ist ein anderes gutes Sterben als wie für Patienten oder Angehörige.“ (E-PF-P-8, 418-433).

$\mathrm{Ob}$ ein Sterbeverlauf friedlich ist oder nicht, hängt nach Aussage einer Pflegeleitung mit dem Thema der Verarbeitung oder Verdrängung einer Krankheit zusammen: Wenn die Krankheit bis zum Schluss weggeschoben wird, ist das für alle Beteiligten, also wohl insbesondere für die Pflegekräfte, ,schwer auszuhalten“. (E-PF-P-4, 205) Wo die bewusste Konfrontation mit der Krankheit bzw. das Annehmen der Krankheit nicht stattfindet, äußert sich das als Unruhe: „Da gab es schon viele Fälle, die wirklich nicht über ihre Krankheit sprechen wollten, weder mit der Familie, noch mit irgendjemand, und die dann einfach extrem unruhig waren und nicht mehr ansprechbar, wo man dann versucht hat, wirklich was Sedierendes und Schmerzmittel zu geben und man viel höhere Dosen brauchte, als bei anderen Patienten, um das zu begleiten." (E-PF-P-4, 228-232) Die Unruhe, die durch diese Situation entsteht, soll durch die Gabe von sedierenden Medikamenten gemildert werden. Unklar bleibt, ob dadurch die Autonomie des Patienten respektiert wird oder nicht. Gutes Sterben bedeutet, eine Krankheit anzunehmen, bedeutet „loslassen“ (E-PFP-9, 224) oder ,,abschließen“ (E-PF-P-9, 372) zu können, etwa indem man das Erbe klärt oder noch einmal Angehörige trifft (E-PF-P-9, 357-363 und 445); nicht gut sterben bedeutet, eine Krankheit komplett zu verdrängen, sie zu verleugnen (E-PFP-9, 247 und 262): „Und Patienten, denen man anmerkt, dass sie nicht bereit sind zu sterben. Dass sie irgendwie, wie ich vorhergesagt habe, noch nicht abgeschlossen haben. Sich noch an irgendwas festhalten. Das finde ich halt, das ist schwierig." (E-PF-P-9, 350-353) Der Sterbeverlauf eines Patienten, der vergeblich auf einen Angehörigen gewartet hat, wird von einer Pflegekraft als ,nicht so rund“ (E-PFH-19, 400) beschrieben, während ein anderer Sterbeprozess rasch zu Ende ging, weil ein Angehöriger anwesend war. Manche sterben, „weil“ ein Angehöriger da 
ist, andere wollen nur für sich sein und sterben, wenn ein Angehöriger gerade weg ist. Als schwierig empfindet es eine Pflegekraft, wenn sich ein schwieriger Sterbeverlauf ,über ewige Zeiten hinweg zieht. Dass man über drei Wochen hinweg stirbt. Weil es doch ein Leiden ist in gewisser Weise." (E-PF-P-9, 447-448) Und ähnlich: „Genauso schlimm finde ich es aber, wenn was sehr, sehr lange und quälend ist. Beispielsweise eben mit Schmerzen, die nicht einstellbar sind, oder Übelkeit oder Ängsten auch ganz schlimm, die nicht einstellbar sind. Oder wenn diese präfinale Phase wahnsinnig lange ist und ja, der Mensch im Grund immer weniger wird, aber überhaupt nicht mehr kontaktierbar ist.“ (E-PF-H-7, 418-422) Allerdings gibt es auch die gegenläufige Haltung, die Patienten zugesteht, ihr Sterben nicht akzeptieren zu wollen: „Und ich akzeptiere deswegen auch, wenn hier Gäste sind, es gibt nämlich auch Gäste, die es nicht akzeptieren wollen bis zum Schluss. Und mich macht das dann wütend, wenn sie das müssen. (...) Aber ich würde mal behaupten, dass es die meisten nicht können. Und dass es da reininterpretiert wird. Dass sie es irgendwie sollen. Sie sollen es können." (E-PF-H-8, 203-210) Zudem besteht die Tendenz, Unruhe zu ,dämpfen“ (E-PF-H-7, 502), obwohl nicht immer klar ist, welche Ursache die Unruhe hat. Ähnlich ein anderer Pfleger: „Und ich glaube inzwischen, dass es einen Sinn hat, dass die Menschen das verdrängen. Weil ich glaube, das wäre sonst nicht aushaltbar.“ (E-PF-H-4, 544-546).

\section{Diskussion}

Bei der Rede vom „guten Sterben“ handelt es sich um eine - normativ gehaltvolle - Semantik. Diese Semantik faltet sich in typischen Merkmalen aus, die - kaum überraschend - in qualitativen wie quantitativen Studien in ähnlicher Weise vorkommen, wobei nicht auszuschließen ist, dass solche Typisierungen den gegenläufigen Weg von der Theorie in die Praxis zurückfinden und sich verfestigen. Bei ihrer Beobachtung kam es uns auch weniger auf die Konvergenzen, als vielmehr auf die Divergenzen an, die wir an Positionsdifferenzen sowohl innerhalb unterschiedlicher als auch zwischen unterschiedlichen Akteursgruppen festmachen. Im Folgenden sollen (1) zunächst typische Merkmale des ,guten Sterbens“ kurz vorgestellt werden. Dann ist (2) der normative Status der beobachteten Typisierungen auch mit Blick auf eingeführte Prinzipien der Medizinethik zu bestimmen. Zuletzt wird (3) überlegt, welche ethisch beachtenswerten Konsequenzen aus diesen Beobachtungen gezogen werden können. Wir lassen uns dabei von der Überzeugung leiten, dass eine Theorie, die von allgemeinen Bestimmungen bzw. von einer allgemeinen Bestimmbarkeit des „guten Sterbens“ ausgeht, möglicherweise die kontextuellen Besonderheiten dieser Rede in ihrer ethischen Relevanz nicht ausreichend in den Blick bekommen kann.

\section{Typische Merkmale}

In unserer Studie haben wir typische Merkmale einer Semantik des ,guten Sterbens" erhoben, die sich, wie schon gesagt, auch in der Forschungsliteratur finden: Idealerweise stirbt ein Patient ruhig, selbstbestimmt, begleitet und gelassen. Diese Aspekte lassen sich genauer bestimmen: (1) Ein ruhiger Sterbeverlauf soll vor al- 
lem durch eine gelungene Symptom- und Schmerzbehandlung über möglichst lange Zeit hinweg erreicht werden, wovon auch das soziale Umfeld profitiert; (2) selbstbestimmt sterben bedeutet, möglichst so viel Bewusstsein zu bewahren, dass jene Bedürfnisse und Wünsche artikuliert werden können, die gestillt bzw. erfüllt werden sollen; (3) begleitet sterben bedeutet, insbesondere in den letzten Augenblicken, jedoch auch in der Zeit davor von Familie, Angehörigen und Freunden sowie Mitarbeitern von Hospizen und Palliativstationen umgeben und umsorgt zu sein und Abschied nehmen zu können; (4) gelassen sterben bedeutet, versöhnt mit sich bzw. dem eigenen Leben sowie insbesondere mit nahe stehenden Menschen zu sein. In den Interviews zeigt sich allerdings auch, dass sich nicht nur typische Vorstellungen von einem guten, sondern auch von einem schlechten Sterben artikulieren, die spezifischer gefasst sind, sich häufig auf konkrete Fälle, die erinnert werden, beziehen und Aufschluss über den Abstand zwischen dem Ideal des guten Sterbens und der Realität geben. Außerdem zeigt sich in seltenen Fällen, dass Patienten einen Zustand für gut erachten, der sich für die soziale Umgebung als schlecht darstellt. Auffällig ist zudem, dass sich Aussagen über das gute bzw. schlechte Sterben weniger bei den Betroffenen selbst, als vielmehr in ihrer Umwelt anlagern, somit Zuschreibungen darstellen: Die Sterbenden kommen über ihr soziales Umfeld zu Wort. Kommen sie selbst zu Wort, wird kaum (oder zumindest nicht so, wie es ihr soziales Umfeld tut) über das (gute oder schlechte) Sterben gesprochen. Ähnliches gilt für die ehrenamtlichen Mitarbeiter sowie für die Angehörigen, so dass über ,gutes bzw. schlechtes Sterben“ fast ausschließlich im professionellen Umfeld gehandelt wird. Dies ist wenig verwunderlich, ist das professionelle Umfeld doch intensiv und dauerhaft mit den unterschiedlichsten Sterbeverläufen konfrontiert, was die Gelegenheit bietet, Erfahrungen zu sammeln und Situationen zu vergleichen.

\section{Ruhiges und selbstbestimmtes Sterben}

Bei Ärzten wie auch bei Pflegekräften ist die Vorstellung eines ruhigen und damit auch guten Sterbens in herausgehobener Weise mit der Symptom- und Schmerzkontrolle verknüpft, wobei die entsprechenden Maßnahmen in der Regel kein kuratives, sondern ein palliatives Ziel verfolgen (sollen). Allgemein wird der Autonomie des Patienten - und dieser Eindruck wird auch in den geführten Interviews bestätigt in der Praxis von Hospizen und Palliativstationen ein hoher Stellenwert zugestanden, gerade weil sie bei nachlassenden mentalen Fähigkeiten nicht (mehr) selbstverständlich ist. Es werden vielerlei Anstrengungen unternommen, um Bedürfnisse und Wünsche der Patienten kennenlernen und stillen bzw. erfüllen zu können. Dennoch wird regelmäßig auf die Spannung zwischen der Ermöglichung eines ruhigen Verlaufs aufgrund einer gelungenen Symptom- und Schmerzkontrolle einerseits und der Ermöglichung eines bewussten und deshalb autonomen Sterbens andererseits hingewiesen. Zwar soll eine Trübung des Bewusstseins möglichst vermieden werden (selbst wenn der Patient dies wünscht), damit Ärzte und Pflegende mit dem Patienten kommunizieren und auf diese Weise seinen Willen erforschen können. In den Interviews wird aber auch geäußert, dass bei einer Schmerzmedikation die Eintrübung des Bewusstseins in Kauf genommen wurde, ohne dass man den Patientenwillen befragt hatte, und zwar in Fällen, in denen vor allem die Unruhe eines 
Sterbenden für die Umwelt schwer erträglich war, sofern sie nämlich durch das Verhalten des Patienten verursacht wurde. Ein ruhiger Sterbeverlauf kommt nicht zuletzt den Ärzten und Pflegekräften, jedoch auch den Angehörigen entgegen. Wird der Sterbeverlauf zur Belastung für das Umfeld, etabliert sich möglicherweise eine autonomieüberstimmende Praxis, die als solche, so vermuten wir, nicht wahrgenommen bzw. reflektiert wird: Patienten werden ruhiggestellt oder zur Einnahme von Medikamenten gedrängt. In einigen Fällen zeigen sich Grauzonen zwischen Fürsorge und Autonomie oder Situationen, die keine eindeutige Zuweisung erkennen lassen, jedoch den Verdacht einer autonomieüberstimmenden Praxis nähren. Moniert wird in den Interviews, dass eine ruhigstellende Medikation eine Routine begründen kann, die dem individuellen Patientenwohl nicht gerecht wird. Insgesamt reichen die Reaktionen der professionellen Akteure vom Aushalten der Unruhe über ein schrittweises Heran- und Zurücktasten in der Symptom- bzw. Schmerzkontrolle, von Versuchen, mit Argumenten zu überzeugen bis zu Versuchen, Patienten zu überreden und schließlich zum Überstimmen des Patientenwillens.

\section{Gelassenes Sterben}

Das Ideal eines gelassenen Sterbens, das uns in den Interviews immer wieder begegnete, ist eng mit dem Ideal des bewussten Sterbens verbunden. Die Vorstellung von einem Loslassen im Sinn einer inneren Auseinandersetzung oder eines Annehmens des eigenen Endes spielt naturgemäß eher bei den Pflegekräften eine Rolle als bei den Ärzten. Ein Patient sollte nicht nur darum wissen, dass alle kurativen Möglichkeiten ausgeschöpft sind und er allenfalls noch palliativ behandelt werden kann; er sollte in diese Art der Therapiebegrenzung auch sichtbar einwilligen, wobei hier nicht die Bekundung des autonomen Willens gemeint ist. Das Ideal scheitert, wenn ein Patient seine Sterberolle erkennbar nicht annimmt, wenn ein Patient kämpft, wenn sich der Sterbeprozess ohne erkennbaren Anlass hinzieht, wenn ein Patient nicht „sterben kann“. Unreflektiert bleibt zumeist, warum ein Mensch gelassen sterben soll bzw. wie sich ein solches Sollen auf den Umgang mit dem Patienten auswirkt. Darüber hinaus etablieren sich soziale und zeitliche Narrative, die normative Wirkungen entfalten, also eine entsprechende Kommunikation auslösen oder die Befindlichkeit insbesondere der Pflegekräfte beinträchtigen: Ein Patient soll noch Abschied nehmen können (oft zitiert wird, dass Sterbende noch auf einen bestimmten Menschen warten, „um“ dann, wenn sie Abschied genommen haben, schnell zu sterben); ein Patient ist unversöhnt und kann deshalb nicht loslassen.

\section{Begleitetes Sterben}

Ähnlich verhält es sich mit dem Ideal, Begleitung im Sterben zu ermöglichen. Hospize und Palliativstationen sehen es als ihre besondere Aufgabe und Herausforderung an, Patienten in ihrer verbleibenden Zeit intensiv zu begleiten. Dies soll durch eine starke, dabei auch rücksichtsvolle Präsenz der Mitarbeiter sowie der Angehörigen geschehen. Diese Präsenz wird auf der Seite der Mitarbeiter als anstrengend empfunden, wenn sie keine offensichtliche Wirkung zeigt, etwa darin, dass Patienten ruhiger werden, dass sie bereitwillig Auskunft über ihre Bedürfnisse und Wünsche 
geben oder sich von Familie und Freunden verabschieden und zufrieden auf ihr Leben zurückschauen können. Wo ein versöhntes Sterben nicht gelingt, muss es von den Menschen, die den Patienten begleiten, ,ausgehalten“ werden. Dies wird besonders in jenen Aussagen deutlich, die ein unbefriedigendes Sterben dort sehen, wo ein Patient unerwartet und deshalb allein, also unbegleitet stirbt. Wo ein solcher begleiteter Abschied vom Leben nicht gelingt, machen es sich die Patienten aus der Sicht der Mitarbeiter nur unnötig schwer, könnte es doch so viel leichter sein - eben auch für die Mitarbeiter.

Insgesamt erscheint uns von unseren Beobachtungen her die Spannung zwischen den Idealen eines selbstbestimmten und eines ruhigen Sterbens dominant zu sein und zu ethisch beachtenswerten Problemen zu führen; die Maßgaben, ein gelassenes oder begleitetes Sterben zu ermöglichen, führen dagegen, auch wo sie Spannungen $\mathrm{zu}$ anderen Idealen sichtbar werden lassen, kaum zu Schwierigkeiten. Im Folgenden soll erörtert werden, wie diese typischen Beschreibungen „eines guten Sterbens“ normativ näher bestimmt werden können.

\section{Der normative Status ,typischer Beschreibungen“}

Generell stützt sich ein Handlungsurteil auf die Wahrnehmung der relevanten Aspekte einer Situation, was in einer entsprechenden Beschreibung mündet, ob dies nun reflexiv geschieht oder nicht. Eine solche Situationsbeschreibung ist notwendig abstrakt, weil bestimmte Merkmale hervorgehoben werden und andere unberücksichtigt bleiben. Nur durch selektive Beschreibungen erhalten Situationen Konturen und werden dadurch auch vergleich- bzw. kommunizierbar, so detailliert sie auch jeweils ausfallen mögen. Die Kommunikation solcher Beschreibungen, auf die sich Handlungsurteile stützen können, dient der Vergewisserung in normativ gehaltvollen Situationen - anderen Personen wie auch sich selbst gegenüber. Zudem verpflichtet die Selektion bestimmter, als relevant erachteter Merkmale dazu, hinreichend ähnliche Situationen annähernd gleich zu behandeln, auch wenn Beschreibungen revidiert werden können, wenn nämlich ein bislang nicht berücksichtigtes Merkmal als relevant erkannt und anerkannt wird (Vieth und Quante 2001, S. 225-228). Auf diese Weise bilden sich normativ gehaltvolle Typisierungen heraus, deren Verpflichtungskraft sich vor der „,kommunikativen Vernunft“ zu bewähren hat: Jeder Akteur hat seine Sicht auf eine bestimmte Situation, ist jedoch auch dazu verpflichtet, sich selbst und anderen gegenüber Rechenschaft darüber zu geben.

Die unterschiedlichen Positionen, die sich in den Interviews über gute bzw. schlechte Sterbeverläufe äußern (das sind Ärzte, Therapeuten, Pflegekräfte, Hospizhelfer, kaum hingegen Patienten bzw. Gäste, ehrenamtliche Mitarbeiter und Angehörige) geben einander in unterschiedlichen Konstellationen Auskunft darüber, was zu tun oder auch zu lassen ist; und sie tun dies, so vermuten wir, weil die Praxis in Hospizen und Palliativstationen im Vergleich zu Krankenhäusern weniger auf standardisierten medizinischen und pflegerischen Handlungen aufruht und die Anzahl eindeutiger Routinen vergleichsweise geringer ist. Das hat auch damit zu tun, dass die kurativen zugunsten der palliativen Maßnahmen und vor allem zugunsten einer intensiven Pflege zurücktreten, die Verweildauer insbesondere in Hospizen extrem kurz sein kann und der Verlauf der Begleitung fast immer mit 
dem Tod des Patienten endet, was in aller Regel gerade eine Zunahme der Hilfsbedürftigkeit bedingt. Für die Akteure besteht dadurch das Bedürfnis, einen Patienten möglichst kennenlernen zu können (was durch die kurze Verweildauer häufig nicht möglich ist), seine Bedürfnisse und Wünsche immer wieder abzuhorchen, auch für den Fall, dass die Fähigkeit zur Kommunikation schwindet oder ganz erlischt. Die dadurch entstehenden Unterbrechungen einer organisatorisch sonst in Fluss gehaltenen Praxis erzeugen zwischen den unterschiedlichen Positionen einen hohen Bedarf an Kommunikation, wodurch zugleich Positionsdifferenzen deutlicher zutage treten: Medizinische und vor allem pflegerische Maßnahmen sollen sich dadurch stärker auf die unterschiedlichen Bedürfnisse und Wünsche sterbender Menschen einstellen können. Daher wird nicht selten betont, dass jeder seinen eigenen Tod stirbt oder dass kein Sterbeverlauf dem anderen gleicht, obwohl, wie gesagt, das Handlungsurteil zur annähernd gleichen Behandlung hinreichend gleicher Fälle verpflichtet. Die Vorstellungen von einer angemessenen Sterbebegleitung können sich situativ verzweigen, jedoch gerade deshalb im Vergleich konsolidiert werden.

Reflektiert man die Rede vom guten Sterben als normativ gehaltvolle Semantik, wird man ihre Funktion in diesem spezifischen Umfeld bestimmen können: Sie dient der kommunikativen Vergewisserung einer sonst vergleichsweise ungewissen Praxis. Werden viele Sterbeverläufe durch das soziale Umfeld als gut beschrieben, erzeugen die selteneren schlechten (zumindest so wahrgenommenen) Sterbeverläufe, wie wir vermuten, einen Erklärungs- und Rechtfertigungsbedarf. Ohnehin ist eine standpunktunabhängige Bewertung von Entscheidungen und Handlungen jenseits bewährter Routinen kaum möglich. Daraus ergibt sich das Erfordernis einer „Neutralisierung“ der Standpunktabhängigkeit des Urteils durch vermehrte Kommunikation. So entstehen in der Praxis der (Selbst-)Vergewisserung bzw. (Selbst-)Rechtfertigung von Entscheidungen und Handlungen kommunikative Verdichtungen: Trotz der schon beschriebenen Individualität der Sterbeverläufe mag daher der Eindruck typischer Merkmale entstehen, die deshalb für eine typische Bewertung eher zur Verfügung stehen können als Merkmale, die den Eindruck von Zufälligkeit oder Einmaligkeit erwecken. Einerseits werden konkrete Bedürfnisse individuell abgehorcht, andererseits zeigt sich das Erfordernis nach einem Vergleich und einer abstrakten Bewertung einzelner Fälle. Dabei haben professionelle Akteure ,ihre“ Bestimmung des ,guten Sterbens“ nicht nur vor sich und dem Team, sondern vor allem vor dem Patienten und seinen Angehörigen zu verantworten.

Würde man normative Orientierungen nur an den Erfordernissen einer Situation ausrichten, wäre zu bezweifeln, dass aus den partikularen Perspektiven überhaupt ein Bedarf an ethischer Klärung entstünde. Es ist, wie schon angedeutet, das Moment des Vergleichs relevanter Merkmale einer Situation, das eine Verpflichtung generiert. Deshalb benötigen die Akteure ein Gespür für die Erfordernisse einer bestimmten Situation. Wenn die Wahrnehmung relevanter Aspekte einer Situation auf normative Erfordernisse aufmerksam macht, liegt das freilich nicht an der Gegebenheit der Situation selbst. Verpflichtungen werden im Vergleich relevanter Merkmale insofern generiert, als sie unter der Voraussetzung ethischer Ansprüche erhoben und von daher überhaupt erst sichtbar werden (Esser 2004, S. 270-272). Ohne die Anerkennung des Prinzips der Autonomie sähe man keinen Anlass, die Entscheidung eines Patienten, die der Empfehlung des Arztes widerspricht, zu respektieren. Entspre- 
chend sind moralische Erfordernisse nicht aus der Beschreibung von Gegebenheiten zu entnehmen. Die Beschreibung einer Situation bringt also nicht eine moralische Forderung hervor, die sich dann in Prinzipien ausdrücken lässt, vielmehr bestimmt der Anspruch ethischer Prinzipien das Erfordernis der jeweiligen Situation. Wir unterscheiden daher situative Forderungen von ethischen Prinzipien.

\section{Ethisch beachtenswerte Konsequenzen}

In unserer empirischen Studie erwies sich das Spektrum typischer Merkmale, das noch feiner ausgefächert werden könnte, zunächst als Ausdruck eines kontextbezogenen Gespürs dafür, in Situationen, in denen nicht auf Routinen zurückgegriffen werden kann, das Richtige zu tun. Gleichzeitig bringt die Ausbildung typischer Merkmale den Effekt mit sich, dass Schwierigkeiten, die bei der Umsetzung der situativen Forderungen auftreten, überhaupt identifiziert und aufgelöst werden können. Dass damit Ideale beschrieben sind, die in der Realität nicht oder kaum je vollständig eingeholt werden können, ist unbestritten. Wir sehen dennoch drei ethisch beachtenswerte Felder.

Der empirische Befund zeigt, was kaum verwundert, (1) dass typische situative Forderungen, die nicht gleichzeitig berücksichtigt werden können, in Konkurrenz oder gar in Konflikt zueinander treten können. (a) Geht es darum, konkurrierende Forderungen einigermaßen in eine Balance zu bringen oder (b) bestimmte Forderungen zeitweilig bevorzugt zu berücksichtigen, auch auf die Gefahr hin, andere vorübergehend in den Hintergrund treten zu lassen, dürfte das Gespür für das situativ richtige Handeln ein ausreichendes Fundament bieten, zumal Ärzte wie Pflegekräfte ein Verständnis für die Standpunktabhängigkeit ihres Entscheidens und Handelns erkennen lassen: Typischerweise balanciert eine gelungene Symptom- bzw. Schmerzbehandlung die Erfordernisse eines ruhigen und eines selbstbestimmten Sterbens gegeneinander aus. Problematischer stellt sich die Situation eines (c) Konflikts verschiedener typischer Forderungen dar, mit der Folge, dass eine Forderung stark oder ganz zurückgedrängt wird. Hier kommt zum Tragen, dass die Verpflichtungskraft situativer Forderungen, zumindest nach unserer Auffassung, nicht ohne Bezug auf ethische Prinzipien denkbar ist. Dies gilt nach unserem Befund vor allem für die situative Forderung, Sterben solle sich selbstbestimmt vollziehen, die sich normativ auf das Prinzip der Autonomie beziehen lässt, doch könnten hier - um einen anerkannten Kanon zu zitieren - auch die Grundsätze des Wohltuns, des Nichtschadens und der Gerechtigkeit angeführt werden (Beauchamp und Childress 1989). Wir sehen in den genannten typischen situativen Forderungen kontextspezifische Bedingungen, zu denen Patienten befähigt bzw. die für sie geschaffen werden sollen (Ermöglichungsbedingungen), während die der Literatur entnommenen Prinzipien Grenzen signalisieren, die die Akteure um den Patienten herum einzuhalten haben (Schutzbedingungen): Sie sollen die Autonomie des Patienten achten, sein Wohl befördern, ihm nicht schaden und ihm Gerechtigkeit widerfahren lassen.

Unterscheiden wir noch präziser zwei Arten von Konflikten: Erstens können situative Forderungen, die ja als berechtigt anerkannt sind, so in Konflikt miteinander geraten, dass die Erfüllung einer Forderung die Erfüllung einer anderen Forderung stark beeinträchtigt oder unmöglich werden lässt. Hier muss gerechtfertigt werden, 
warum eine Forderung gegenüber einer anderen deutlich bevorzugt werden soll: Ein Patient verhält sich unruhig und wird deshalb sediert, was sein Bewusstsein und seine Selbstbestimmheit beeinträchtigt. Zweitens kann es innerhalb einer solchen Praxis des Bevorzugens situativer Forderungen auch zum Konflikt mit einem ethischen Prinzip kommen: Ein Patient will ein klares Bewusstsein behalten und wird dennoch sediert; oder ein Patient will den Verlauf seines Sterbens nicht bewusst erleben, wird jedoch aus diesem Grund nicht sediert (vorausgesetzt sei hier der Einfachheit halber, dass eine Sedierung mit dem Ziel, das Bewusstsein zu vermindern oder auszuschalten, legitim sein kann, etwa wenn psychisches Leid vorliegt). Wir gehen dabei, wie bereits bemerkt, davon aus, dass situative Forderungen in normativer Hinsicht von ethischen Prinzipien abhängig sind, ohne sie also keine Verpflichtungskraft entfalten können, was kasuistische Ansätze bestreiten würden. Deshalb plädieren wir für einen prinzipienbasierten Ansatz, der freilich einem situativen Gespür Raum gibt, nämlich dort, wo Prinzipien der Spezifizität konkreter Fälle nicht gerecht werden. Der Rechtfertigungsbedarf liegt somit darin, eine im jeweiligen Kontext intuitiv plausible Hierarchie von konkurrierenden oder konfligierenden situativen Forderungen zu bilden, ohne ein ethisches Prinzip zu verletzen.

So ist es beispielsweise denkbar, dass die Autonomie eines Patienten aktual eingeschränkt wird, um Pflegekräften unzumutbare Situationen zu ersparen, jedenfalls wenn dies durch eine autonome Entscheidung des Patienten gedeckt ist. Steht etwa die Forderung, ein selbstbestimmtes Sterben zu ermöglichen, mit der Forderung, ein ruhiges, friedliches und gelassenes Sterben zu fördern, in Konkurrenz oder Konflikt, ist somit eine Beschränkung der Fähigkeit, selbstbestimmt zu entscheiden und zu handeln, nur dann legitim, wenn dies auch dem Willen des Patienten entspricht und so dem Prinzip der Autonomie Genüge getan wird (die Diskussion darüber, was als ein solcher Wille gelten kann und wie er in Erfahrung zu bringen ist, kann hier nicht geführt werden). Wären mehrere ethische Prinzipien verletzt, stünden also ethische Prinzipien in Konflikt zueinander, hielte zumindest ein pluralistisches Modell keine Regel für die Lösung eines solchen Konflikts bereit. Solange der Patient autonomiefähig ist, darf das Prinzip der Autonomie jedoch nicht anderen Prinzipien untergeordnet werden. In dieser Hinsicht legt es sich, auch wenn man einen Prinzipienpluralismus vertritt, nahe, dem Prinzip der Autonomie eine besondere Stellung vor anderen ethischen Prinzipien zuzusprechen. Den Interviews lässt sich nicht eindeutig entnehmen, ob die Autonomie eines Patienten tatsächlich überstimmt wurde. Wir formulieren deshalb hypothetisch und ethisch nicht trivial: Würde die Autonomie eines Patienten überstimmt, könnten die sonst beachtenswerten Forderungen, insbesondere die Forderung, ein ruhiges Sterben zu ermöglichen, keine legitimen Gründe für eine solche autonomieüberstimmende Praxis liefern. Die Forderungen, ein gelassenes bzw. ein begleitetes Abschiednehmen zu ermöglichen, standen dagegen nach unserer Beobachtung, wie schon angedeutet, weder mit anderen Forderungen noch mit einem ethischen Prinzip in Konflikt.

Daneben scheint uns (2) bemerkenswert, dass - womöglich auch kollektiv bestätigte - Beschreibungen in Wirklichkeit wunschgeleitete Deutungen (Esser 2004, S. 293-298) darstellen können: Hinter einem Suizidwunsch verberge sich, so wurde etwa angeführt, in Wirklichkeit die Angst eines Patienten vor unerträglichen Schmerzen oder davor, ohne Begleitung sterben zu müssen. Dies führte zu dem Ur- 
teil, eine palliative Sedierung dürfe nicht einfach das Bewusstsein vermindern oder auslöschen, nur weil ein Patient sein Sterben nicht bewusst mitverfolgen will. Sehen wir von der ethischen und rechtlichen Diskussion ab, hätte eine solche Deutung die Konsequenz, dass ein möglicherweise legitimer Wunsch nicht erfüllt wird. Hier müsste wieder auf das Verhältnis von situativen Forderungen und ethischen Prinzipien Bezug genommen werden. Auch der häufig vorgebrachte Hinweis, eine Schmerzbzw. Symptomkontrolle sei die Voraussetzung für einen ruhigen Sterbeverlauf, wird durch Pflegekräfte gelegentlich kritisiert: Die Unruhe eines Patienten könne auch andere Ursachen haben und auf andere Bedürfnisse verweisen. Wird dies übersehen, entstehe eine routinierte Behandlung, die an den Bedürfnissen eines Patienten vorbeigehen mag. Auffallend ist in diesem Zusammenhang, dass die psychische Verfassung der Patienten durch Ärzte weit weniger als die physische Verfassung mit dem guten bzw. schlechten Sterben in Verbindung gebracht wird. Auch spirituelles bzw. existenzielles Leid wird kaum thematisiert. Weniger problematisch erscheinen deutende Beschreibungen, die in unseren Augen Narrative bilden, sofern sie keine Handlungsgründe bereitstellen sollen: Patienten sterben so, wie sie gelebt haben; Patienten sterben zu schnell oder zu langsam, erst in Anwesen- oder erst in Abwesenheit einer Bezugsperson, je nach dem Anschein des Krankheitsverlaufs. Dennoch können solche Narrative zu verzerrenden Situationsbewertungen führen. Nur in seltenen Fällen wird von professionellen Akteuren gut genannt (die Schmerzen eines Patienten sollen reduziert werden), was ein Patient als schlecht empfindet (er will die Schmerzen bewusst aushalten). Hier zeigt sich (anders als bei der Konkurrenz oder beim Konflikt von situativen Forderungen) erneut die Standpunktabhängigkeit eines Urteils, die zu unterschiedlichen Deutungen führen kann. Ethisch gesehen ist das Prinzip der Autonomie ausschlaggebend, das die Deutungshoheit so weit und so lange als möglich dem Patienten zugesteht.

Schließlich wird (3) gelegentlich nicht reflektiert, dass die Bedingungen, unter denen die Betreuung Sterbender stattfindet, Gewordenheiten, nicht Gegebenheiten darstellen und insofern veränderbar sind. Dies wird in Situationen deutlich, in denen auf (durchaus berechtigte) Bedürfnisse von Ärzten, Pflegekräften oder Angehörigen verwiesen wird, Situationen, die die Praxis beeinflussen, bis dahin, dass sie zur Überstimmung des Patientenwillens führen können. Wenn wahr sein oder wahr werden soll, dass jeder seinen eigenen Tod stirbt (wie oft gesagt wird), müsste diese Individualität zumindest vom sozialen Umfeld des Sterbenden zugestanden werden. Weil niemand ohne soziale Bezüge stirbt (schon gar nicht in Hospizen oder Palliativstationen), selbst wenn sein Leben einsam zu Ende gehen sollte (oder auch nur: wenn niemand dabei ist), ist die Rede vom ,,je eigenen Tod“ streng genommen eine unerfüllbare Illusion, spielen dabei doch die Bedürfnisse und Wünsche vieler Menschen eine Rolle, Bedürfnisse und Wünsche, die nicht einfach abgewiesen werden können und die, im Gegenteil, dann in Erscheinung treten, wenn sie nicht auf „einen Nenner“ zu bringen sind. So berechtigt diese Bedürfnisse freilich auch sein mögen, sie dürfen einen ethisch problematischen Konflikt nicht verdecken. Eher wäre zu überlegen, wie solche Konflikte strukturell (also über Regeln, Personal oder Ausstattung) aufgelöst werden können. Sind Pflegekräfte mit einer Situation überfordert, ist es eine Option, den Patienten (möglicherweise autonomieüberstimmend) ruhigzustellen, eine andere, die Betreuungssituation zu verändern. Uns ist klar, dass 
hier Ideal und Realität in Spannung stehen, doch ist es Ziel der Ethik, die Realität, wo möglich, zu verbessern und entsprechende Empfehlungen zu geben.

Funding Open Access funding provided by Projekt DEAL.

Open Access Dieser Artikel wird unter der Creative Commons Namensnennung 4.0 International Lizenz veröffentlicht, welche die Nutzung, Vervielfältigung, Bearbeitung, Verbreitung und Wiedergabe in jeglichem Medium und Format erlaubt, sofern Sie den/die ursprünglichen Autor(en) und die Quelle ordnungsgemäß nennen, einen Link zur Creative Commons Lizenz beifügen und angeben, ob Änderungen vorgenommen wurden.

Die in diesem Artikel enthaltenen Bilder und sonstiges Drittmaterial unterliegen ebenfalls der genannten Creative Commons Lizenz, sofern sich aus der Abbildungslegende nichts anderes ergibt. Sofern das betreffende Material nicht unter der genannten Creative Commons Lizenz steht und die betreffende Handlung nicht nach gesetzlichen Vorschriften erlaubt ist, ist für die oben aufgeführten Weiterverwendungen des Materials die Einwilligung des jeweiligen Rechteinhabers einzuholen.

Weitere Details zur Lizenz entnehmen Sie bitte der Lizenzinformation auf http://creativecommons.org/ licenses/by/4.0/deed.de.

\section{Einhaltung ethischer Richtlinien}

Interessenkonflikt C. Breitsameter gibt an, dass kein Interessenkonflikt besteht.

Ethische Standards Das Projekt inkl. der Befragungen wurde von der zuständigen Ethikkommission beraten und im Einklang mit nationalem Recht sowie gemäß der Deklaration von Helsinki von 1975 (in der aktuellen, überarbeiteten Fassung) durchgeführt. Von allen beteiligten Probanden liegt eine Einverständniserklärung vor.

\section{Literatur}

Beauchamp TL, Childress JF (1989) Principles of biomedical ethics, 3. Aufl. Oxford University Press, Oxford

Corbin J, Strauss AL (2008) Basics of qualitative research. SAGE, Thousand Oaks

Esser A (2004) Eine Ethik für Endliche. Kants Tugendlehre in der Gegenwart. Frommann-Holzboog, Stuttgart, Bad Cannstatt

Glaser BG, Strauss AL (1967) The discovery of grounded theory: Strategies of qualitative research. Aldine, Chicago

Göckenjan G (2008) Sterben in unserer Gesellschaft - Ideale und Wirklichkeiten. Polit Zeitgesch 18:7-14

Göckenjan G, Dreßke S (2002) Wandlungen des Sterbens im Krankenhaus und die Konflikte zwischen Krankenrolle und Sterberolle. Österr Z Soziol 27:80-96

Granda-Cameron C, Houldin A (2012) Concept analysis of good death in terminally ill patients. Am J Hosp Palliat Care 29:632-639

Mak JMH, Clinton M (1999) Promoting a good death: an agenda for outcomes research - A review of the literature. Nurs Ethics 6:97-106

Miyashita M et al (2007) Good death in cancer care: a nationwide quantitative study. Ann Oncol 18:1090-1097

Morell EA (2012) Learning that a death can be a good death. J Palliat Med 15:248-249

Seale C, Addington-Hall J, McCarthy M (1997) Awareness of dying: prevalence, causes and consequences. Soc Sci Med 45:477-484

Steinhauser K et al (2000) In search of a good death: observations of patients, families, and providers. Ann Intern Med 132:825-832

Vieth A, Quante M (2001) Wahrnehmung oder Rechtfertigung? Zum Verhältnis inferenzieller und nichtinferenzieller Erkenntnis in der partikularistischen Ethik. Jahrb Wiss Ethik 6:203-234

Walters G (2004) Is there such a thing as a good death? Palliat Med 18:404-408 